\title{
ESTRATÉGIAS PARA CAPACITAÇÃO AO CUIDADO EM TUBERCULOSE
}

\author{
Sonaly Melo de Macedo ${ }^{1}$, Rayla Patrícia da Silva Andrade ${ }^{1}$, Cíntia Raquel Batista de Andrade Souza ${ }^{1}$, Anny \\ Suelen dos Santos Andrade ${ }^{1}$, Tereza Cristina Scatena Villa ${ }^{2}$, Érika Simone Galvão Pinto ${ }^{3}$
}

\begin{abstract}
RESUMO: Pesquisa quantitativa que objetivou descrever os componentes ligados à capacitação em tuberculose que tornam as equipes de saúde capazes de identificar estratégias de cuidado. Aplicou-se um questionário estruturado com 100 profissionais em 27 Unidades de Saúde no município de Natal, estado do Rio Grande do Norte, entre novembro de 2013 e janeiro de 2014. Os dados foram analisados pelo programa estatístico Statistical Package for Social Sciences versão 22.0. Os resultados mostraram que 60\% $(n=60)$ dos profissionais utilizaram frequentemente o manual de diretrizes para o controle da tuberculose integrando a prática cotidiana; $73 \%$ $(\mathrm{n}=73)$ afirmaram que o envolvimento de especialistas em tuberculose é realizado por meio de referenciamento; $68 \%(n=68)$ repassam as informações sobre tuberculose a todos os usuários e $44 \%(n=44)$ das capacitações são realizadas esporadicamente. Conclui-se que há a necessidade de investimento em capacitação sistemática envolvendo também especialistas, visando o manejo e controle da tuberculose.
\end{abstract}

DESCRITORES: Capacitação em serviço; Tuberculose; Atenção Primária à saúde; Educação em saúde; Assistência ao paciente.

\section{STRATEGIES FOR TUBERCULOSIS CARE TRAINING}

ABSTRACT: Quantitative study that aimed to describe the components related to tuberculosis training that make health teams able to identify care strategies.A structured questionnaire was applied with 100 professionals in 27 Health Units of Natal, Rio Grande do Norte, between November 2013 and January 2014. The data were analyzed using the Statistical Package for the Social Sciencesversion 22.0. The results showed that $60 \%(n=60)$ of the professionals often used the guidelines for the control of tuberculosis manual in the everyday practice; $73 \%(n=73)$ stated that the involvement of tuberculosis specialists is accomplished by referral; $68 \%(n=68)$ pass on the information about TB to all users and $44 \%(n=44)$ of the training programs are carried out sporadically.lt was concluded that there is the need to invest in systematic training involving specialists, aimed at the management and control of tuberculosis.

DESCRIPTORS: In service training; Tuberculosis; Primary healthcare; Health education; Patient care.

\section{ESTRATEGIAS PARA CAPACITACIÓN AL CUIDADO EN TUBERCULOSIS}

RESUMEN: Investigación cuantitativa con objeto de describir los componentes vinculados a la capacitación en tuberculosis que hacen los equipos de salud capaces de identificar estrategias de cuidado. Fue aplicado un cuestionario estructurado a 100 profesionales en 27 Unidades de Salud en el municipio de Natal, estado de Rio Grande do Norte, Brasil, entre noviembre del 2013 y enero del 2014. Los datos fueron analizados en el programa estadístico Statistical Package for Social Sciences versión 22.0. Los resultados mostraron que $60 \%(n=60)$ de los profesionales utilizaron frecuentemente el manual de directivas para el control de la tuberculosis integrando la práctica cotidiana; $73 \%(n=73)$ afirmaron que el involucramiento de especialistas en tuberculosis se hace mediante referencia; $68 \%(n=68)$ transmiten las informaciones sobre tuberculosis a todos los usuarios y $44 \%$ ( $n=44)$ de las capacitaciones ocurren esporádicamente. Se concluye que son necesarias inversiones en capacitación sistemática, involucrando también especialistas, visando al manoseo y control de la tuberculosis.

DESCRIPTORES: Capacitación en servicio; Tuberculosis; Atención Primaria de salud: Educación en salud; Atención al paciente.

${ }^{1}$ Discente de Enfermagem. Universidade Federal do Rio Grande do Norte. Natal, RN, Brasil.

${ }^{2}$ Enfermeira. Doutora em Enfermagem. Docente da Escola de Enfermagem de Ribeirão Preto da Universidade de São Paulo. Ribeirão Preto, SP, Brasil.

${ }^{3}$ Enfermeira. Doutora em Enfermagem. Docente de Enfermagem da Universidade Federal do Rio Grande do Norte. Natal, RN, Brasil.

Autor Correspondente:

Recebido: 22/02/2016

Sonaly Melo de Macedo

Finalizado: 21/09/2016

Universidade Federal do Rio Grande do Norte

Av. Senador Salgado Filho, 3000 - 59078-970 - Natal, RN, Brasil

Email: sonaly_melo@yahoo.com.br 


\section{INTRODUÇÃO}

A capacitação profissional constitui uma ferramenta importante para o manejo e controle da Tuberculose (TB), auxiliando os profissionais de saúde na assistência ao paciente e na detecção precoce da doença.

Atualmente, torna-se inquestionável a necessidade sentida pelos serviços de saúde em capacitar seus profissionais. Na prática cotidiana observam-se dificuldades enfrentadas para intervir de maneira eficaz frente aos problemas de saúde da comunidade ${ }^{(1)}$.

Capacitar significa garantir a utilização das habilidades de um profissional e dessa forma potencializar seu conhecimento para lidar com determinada situação. Porém, a capacitação profissional ocorre esporadicamente nos serviços e na maioria das vezes ocorre juntamente com o trabalho, o que resulta na ausência de disponibilidade de tempo e motivação para a realização dessa atividade. Assim, a educação continuada é uma estratégia necessária que os serviços devem dispor para treinar e capacitar os funcionários, pois além de motivar na busca pela qualificação, dá oportunidade de desenvolvimento profissional e pessoal ${ }^{(2)}$.

O investimento na capacitação dos trabalhadores pode garantir o êxito no planejamento das ações desenvolvidas na promoção ou restabelecimento de saúde da população.

Quando se trata da TB, a capacitação profissional consiste em garantir a compreensão da doença e de seus condicionantes, a prevenção, a identificação precoce dos casos, o controle da doença incluindo o tratamento supervisionado e a baciloscopia dos sintomáticos respiratórios $(\mathrm{SR})^{(1,3-4)}$.

No campo da TB, os profissionais de saúde precisam estar atentos aos SR, tendo em vista ainda o acometimento desta patologia em milhares de pessoas, constituindo um problema de saúde pública. Apesar de estratégias para o controle da doença, ainda encontram-se elevadas taxas de incidência e mortalidade ${ }^{(5)}$. O Brasil representa um dos 22 países priorizados pela Organização Mundial de Saúde (OMS), tendo como característica a concentração de $80 \%$ da carga mundial de TB com estimativa de 50 milhões de infectados, 109.672 casos novos e 6000 óbitos ao ano. Em 2012, cerca de 8,6 milhões de pessoas desenvolveram a TB e 1,3 milhões morreram, incluindo 320.000 mortes com o Vírus da Imunodeficiência Humana (HIV) positivas, sendo a maioria homens ${ }^{(6)}$.

O Brasil diagnosticou 71.123 casos novos de TB em 2013, perfazendo um coeficiente de incidência de 35,4/100.000 habitantes e um coeficiente de mortalidade de 2,3/100.000 habitantes $^{(7)}$.

Para melhorar os indicadores epidemiológicos e operacionais em TB, é fundamental investir nas capacitações periódicas, o que pode contribuir para o manejo e controle dessa doença. Porém, há outros fatores envolvidos, dentre eles o compromisso de cada profissional ${ }^{(8)}$ e o interesse do paciente em cooperar com o seu tratamento. Objetivou-se com este estudo descrever os componentes ligados à capacitação em TB, os quais tornam as equipes de saúde capazes de identificar estratégias de cuidado.

\section{- METODOlOGiA}

Trata-se de uma pesquisa de delineamento descritivo e abordagem quantitativa, realizada no município de Natal, estado do Rio Grande do Norte. A população estimada do município é de 862.044 habitantes ${ }^{(9)}$, possui uma cobertura de $50 \%$ da Estratégia de Saúde da Família (ESF) e conta com 60 Unidades de Saúde, sendo 23 Unidades Básicas de Saúde (UBS) e 37 Unidades de Saúde da Família (USF) distribuídas em cinco Distritos Sanitários (DS): Norte I e II, Sul, Leste e Oeste ${ }^{(10)}$.

Em relação a TB, o município no ano de 2013 obteve: 56,9\% de cura, 28,7\% de Tratamento Diretamente Observado (TDO), 48,4\% de teste anti HIV realizado, entre os casos de TB, e uma incidência de $57 / 100.000$ habitantes $^{(11)}$.

Foi realizado um levantamento junto à Secretaria Municipal de Saúde de Natal (SMS) para a obtenção do quantitativo de recursos humanos por categoria profissional: médico, enfermeiro, auxiliar de enfermagem e/ou técnico em enfermagem e Agente Comunitário de Saúde (ACS) que atuam na 
Atenção Primária à Saúde (APS) e já acompanharam casos de TB, totalizando 384 profissionais. Utilizouse uma amostra aleatória, considerando P (Proporção populacional $)=0,5$, IC (Intervalo de confiança) $=$ $95 \%$ e um erro amostral de $5 \%$ totalizando um $n=100$ profissionais de saúde da APS.

Como critérios de inclusão foram estabelecidos: profissionais de saúde atuantes no período da coleta de dados, que já acompanharam casos de TB durante o tratamento. Não foram previstos critérios de exclusão.

Em cada Unidade de Saúde foram escolhidos quatro profissionais de saúde, sendo um por categoria: médico, enfermeiro, técnico em enfermagem e ACS. Havendo recusa de algum profissional, este foi substituído até completar o $\mathrm{n}=100$ profissionais de saúde. Obteve-se 32 recusas, sendo 17 médicos, um enfermeiro, 12 técnicos em enfermagem e dois ACS.

Aplicou-se um questionário estruturado em 27 Unidades, entre novembro de 2013 e janeiro de 2014. A escolha das Unidades deu-se a partir de sorteio aleatório considerando aquelas em que os profissionais já tinham acompanhados casos de TB. Aqueles que aceitaram participar foram informados pelos pesquisadores quanto à pesquisa e os objetivos do estudo, mediante assinatura do Termo de Consentimento Livre e Esclarecido. A coleta de dados foi realizada em local privado na Unidade de Saúde.

O instrumento utilizado foi proposto por Mac Coll Institute for Health Care Innovation; adaptado e validado no Brasil(12) para a "avaliação pelos profissionais da capacidade institucional local para desenvolver o modelo de atenção às condições crônicas" para atenção à TB. Está dividido em 07 dimensões: I- Organização da atenção à tuberculose; II- Articulação com a comunidade; IIIAutocuidado apoiado; IV- Suporte à decisão; V- Desenho do sistema de prestação de serviços; VISistema de informação clínica; VII- Integração dos componentes do modelo de atenção às pessoas com TB. O foco desse estudo é a dimensão IV, composta por 04 componentes: manual de diretrizes para o controle da TB na Unidade de Saúde; envolvimento de especialistas em TB que não faziam parte da equipe no apoio aos profissionais de saúde da Unidade; capacitação dos profissionais de saúde para atenção à TB; e informação às pessoas sobre TB.

A obtenção das respostas foi classificada em 04 níveis (D, C, B, A) que foram representados por valores que variaram de 0 a 11. Sendo D correspondente ao nível mais desfavorável, com pontuação de 0 a 2; C, de 3 a 5, e B, de 6 a 8, sendo esses dois níveis intermediários; e A, o mais favorável, de 9 a 11.

A interpretação dos resultados deu-se da seguinte forma: capacidade limitada para a atenção aos portadores de TB, com pontuações de 0 a 2; capacidade básica de 3 a 5; capacidade razoável de 6 a 8; e capacidade ótima de 9 a 11. Calculado a partir da média aritmética entre o somatório das respostas da dimensão, dividido pela quantidade total de componentes.

Os dados foram analisados pelo programa estatístico Statistical Package for Social Sciences (SPSS) utilizando a versão 22.0.

O projeto de pesquisa foi aprovado pelo Comitê de Ética em Pesquisa da Universidade Federal do Rio Grande do Norte, com número do parecer: 456.332, data da relatoria: 01/11/2013 e CAAE: 18751132.1001.5537, atendendo à resolução 466/12 do Conselho Nacional de Saúde(13).

\section{RESUltados}

Nesse estudo participaram 100 profissionais: 35 enfermeiros, 22 técnicos em enfermagem, nove médicos e 34 ACS. Das entrevistas realizadas, $80 \%(n=80)$ foram respondidas por profissionais de USF, $18 \%(n=18)$ de UBS e duas (2\%) de Unidade Mista. Verificou-se que $41 \%(n=41)$ trabalham na Unidade há mais de 10 anos, 29\% ( $n=29)$ trabalham de 6 a 10 anos, 23\% $(n=23)$ de 1 a 5 anos e sete $(7 \%)$ há menos de um ano. Quanto à categoria profissional, 79\% $(\mathrm{n}=79)$ dos ACS trabalham há mais de 10 anos, 14\% $(n=14)$ dos médicos de 6 a 10 anos, quatro (4\%) dos técnicos em enfermagem de 1 a 5 anos e três $(3 \%)$ dos enfermeiros há menos de um ano.

Observou-se, entre os 100 participantes, que a maioria referiu uma capacitação básica e razoável 46\% $(n=46)$, no que diz respeito à atenção aos portadores de TB no que envolve o cuidado e o tratamento da doença, conforme dados mostrados na Tabela 1. 
Tabela 1 - Julgamento realizado pelos profissionais da Atenção Primária à Saúde segundo o cuidado prestado aos portadores de tuberculose. Natal, RN, Brasil, 2014

\begin{tabular}{lcc}
$\begin{array}{l}\text { Julgamento realizado pelos profissionais da Atenção Primaria à Saúde } \\
\text { segundo o cuidado prestado aos portadores de tuberculose }\end{array}$ \\
\hline Capacidade & $\mathbf{n}$ & $\%$ \\
\hline Limitada & 3 & 3 \\
\hline Básica & 46 & 46 \\
\hline Razoável & 46 & 46 \\
\hline Ótima & 5 & 5 \\
\hline Total & 100 & 100
\end{tabular}

Quanto à classificação dos componentes ligados à capacitação em TB que tornam as equipes de saúde capazes de identificar estratégias de cuidado, os dados apresentados na Tabela 2 mostraram que os componentes que atingiram capacidade ótima foram: manual de diretrizes para o controle da TB na Unidade de Saúde, 60\% (n=68), e informações às pessoas sobre TB, 68\% (n=68); e capacidade limitada: envolvimento de especialistas em TB que não faziam parte da equipe no apoio aos profissionais de saúde da Unidade, 73\% ( $n=73)$, e capacitação dos profissionais de saúde para atenção à TB, 44\% $(n=44)$.

Em relação ao manual de diretrizes para o controle da TB, $60 \%(n=60)$ dos profissionais consideraram a capacidade como sendo ótima, estando este disponível na Unidade e utilizado frequentemente pelos profissionais da saúde, sendo integrado à prática cotidiana na atenção à TB, e oito (8\%) atribuíram como limitada, ou seja, o manual não estava disponível, levando-se a crer que os profissionais faziam uso dele para fornecer as informações sobre TB aos pacientes.

No que se refere ao envolvimento de especialistas em TB que não faziam parte da equipe no apoio aos profissionais de saúde da Unidade, $73 \%(n=73)$ dos profissionais classificaram como capacidade limitada, com o contato com o especialista por meio de referenciamento/ encaminhamento tradicional. Três (3\%) afirmaram ter capacidade ótima, por meio da participação periódica de especialistas que auxiliavam no processo de educação permanente e discutiam casos com a equipe.

Com relação à capacitação dos profissionais de saúde para a atenção à TB, 44\% (n=44) afirmaram ter capacidade limitada, ou seja, a capacitação era realizada esporadicamente. Apenas $12 \%(n=12)$ relataram possuir capacidade ótima, com capacitações realizadas sistematicamente, através de métodos educacionais que promoviam efetiva incorporação de ações de controle da TB à prática diária da Unidade de Saúde, incluindo toda a equipe de profissionais.

Quanto à informação prestada aos indivíduos sobre TB, 68\% $(n=68)$ dos profissionais classificaram como capacidade ótima, sendo passadas para todos os usuários, e incluíam o papel de cada indivíduo na prevenção e tratamento da TB. Enquanto que 10\% $(n=10)$ afirmaram ter capacidade básica, realizada apenas quando os portadores de TB solicitavam, por meio de informações escritas (folder, cartazes, etc.).

Tabela 2 - Classificação dos componentes ligados à capacitação em tuberculose que tornam as equipes de saúde capazes de identificar estratégias de cuidado. Natal, RN, Brasil, 2014

\begin{tabular}{|c|c|c|c|c|c|c|c|c|c|}
\hline \multirow[t]{2}{*}{ Componentes } & \multicolumn{2}{|c|}{$\begin{array}{l}\text { Capacidade } \\
\text { Limitada } \\
\end{array}$} & \multicolumn{2}{|c|}{$\begin{array}{l}\text { Capacidade } \\
\text { Básica }\end{array}$} & \multicolumn{2}{|c|}{$\begin{array}{l}\text { Capacidade } \\
\text { Razoável }\end{array}$} & \multicolumn{2}{|c|}{$\begin{array}{c}\text { Capacidade } \\
\text { Ótima }\end{array}$} & \multirow[t]{2}{*}{ Total } \\
\hline & $\mathbf{n}$ & $\%$ & $\mathbf{n}$ & $\%$ & $\mathbf{n}$ & $\%$ & $\mathbf{n}$ & $\%$ & \\
\hline $\begin{array}{l}\text { Manual de diretrizes para o controle da } \\
\text { TB* }\end{array}$ & 8 & 8 & 9 & 9 & 23 & 23 & 60 & 60 & 100100 \\
\hline $\begin{array}{l}\text { Envolvimento de especialistas em } \mathrm{TB}^{*} \text { que } \\
\text { não faziam parte da equipe }\end{array}$ & 73 & 73 & 20 & 20 & 4 & 4 & 3 & 3 & 100100 \\
\hline $\begin{array}{l}\text { Capacitação dos profissionais de saúde } \\
\text { para a atenção à TB* }\end{array}$ & 44 & 44 & 16 & 16 & 28 & 28 & 12 & 12 & 100100 \\
\hline Informação às pessoas sobre TB* & 11 & 11 & 10 & 10 & 11 & 11 & 68 & 68 & 100100 \\
\hline
\end{tabular}

TB*- Tuberculose 


\section{- DISCUSSÃO}

O tempo de atuação dos profissionais no serviço de saúde constitui ferramenta importante para fortalecer o vínculo e a confiança com os usuários. A maioria dos profissionais atuantes na APS está no serviço há mais de 10 anos, semelhante aos resultados de uma pesquisa ${ }^{(14)}$. Quanto à categoria profissional, verificou-se que os ACS eram os profissionais que trabalhavam há mais tempo exercendo sua função.

A rotatividade dos profissionais nos serviços de saúde influencia no tempo de serviço, o que se observa através do rompimento na manutenção das equipes já qualificadas num determinado serviço de saúde ${ }^{(15)}$, o que pode comprometer o acompanhamento sistemático do portador de TB. O tempo de trabalho na unidade, juntamente com a qualificação profissional, podem favorecer o diagnóstico precoce e consequentemente a cura do indivíduo.

O que interfere na incorporação de ações de controle da TB é a qualificação dos profissionais, pois muitos passam a se responsabilizar por programas que nunca assumiram ${ }^{(3)}$.

A maioria dos profissionais tem conhecimento sobre as atividades do Programa Nacional de Controle da Tuberculose (PNCT) ${ }^{(16)}$ que são: cadastro do usuário, planejamento de atividades, reuniões de equipe, relatório das atividades, análise do relatório e supervisão de atividades. Um estudo mostrou falta de conhecimento na supervisão de atividades ${ }^{(17)}$. Porém, ausência de capacitação, demora nos resultados laboratoriais, ausência de médicos especialistas e enfermeiros que se dediquem ao PNCT, a falta de materiais e demora no agendamento de exames, estão ocorrendo também nas redes de apoio para os pacientes, impedindo assim a conclusão do tratamento ambulatorial ${ }^{(18)}$.

Além das pessoas não saberem identificar os sinais e sintomas da TB, os profissionais não são preparados, dificultando dessa forma um diagnóstico correto e o início do tratamento o mais breve possível, evitando assim a propagação da doença ${ }^{(19)}$.

Neste estudo pode-se verificar que a capacitação profissional ainda precisa ser melhorada, para atingir nível de capacidade ótima para diagnosticar e tratar os doentes.

Segundo dados apresentados em uma pesquisa, constatou-se que $86 \%(n=286)$ dos profissionais eram capacitados, mas apenas 69,5\% (n=286) tinham capacitação suficiente para realizar ações de controle da $\mathrm{TB}^{(4)}$, o que corrobora com este estudo.

O conhecimento adquirido na graduação não é o suficiente para detectar precocemente a TB e poder intervir efetivamente no controle da doença ${ }^{(1)}$. A ineficácia de informações, por parte dos profissionais de saúde, acerca do modo de transmissão, sinais e sintomas contribui para o retardo do diagnóstico da doença(5). Assim, há necessidade de profissionais e gestores reconhecerem que a capacitação em saúde se faz necessária nos serviços ${ }^{(1)}$.

Nesta pesquisa observou-se que os profissionais de saúde classificaram a capacitação profissional como limitada, porém a maioria fazia uso do manual de diretrizes para o controle da TB.

Autores das ciências sociais referem que quando se segue um protocolo clínico, torna-se mais fácil atingir os resultados e os objetivos estabelecidos, afinal há melhor controle de todas as atividades e ações que serão realizadas no serviço ${ }^{(17)}$.

Vale salientar que as Unidades de Saúde não devem apenas seguir as rotinas estabelecidas no serviço. É importante que os profissionais de saúde estejam mais acessíveis à realidade epidemiológica e social do território, rompendo com a lógica programática de instauração de necessidades ${ }^{(20)}$.

Observa-se no cotidiano da prática profissional, relacionado às ações de TB, déficit quanto ao envolvimento de especialistas no decorrer do tratamento e o acúmulo de atividades do enfermeiro.

De acordo com uma pesquisa realizada com 81 doentes, observou-se que $98,77 \%$ ( $n=80$ ) nunca eram encaminhados aos especialistas pelos profissionais de saúde e nem havia discussão dos possíveis lugares de atendimento. O estudo ainda relata que 94,05\% $(n=79)$ dos 84 profissionais de saúde nunca discutiam os resultados da consulta com especialistas, como também não procuravam saber como foi o seu atendimento ${ }^{(21)}$. Isso leva a crer que o envolvimento de especialistas no controle da TB encontra- 
se limitado, podendo estar relacionado à escassez de recurso humano, como também do próprio envolvimento entre os profissionais de saúde.

Os estabelecimentos que dispõem de pessoal treinado e capacitado, com ações educativas voltadas à TB, possivelmente apresentarão melhor compreensão desta doença e dos seus condicionantes, podendo assim diagnosticar, tratar, prevenir e controlá-la. Também é importante a corresponsabilização do doente no processo saúde-doença, pois a cura não depende somente do profissional que o acompanha.

A falta de ações educativas em relação à TB e seu tratamento fazem com que o doente não se motive para buscar mudanças para promover a sua saúde. No entanto, as equipes de saúde devem incluir ações educativas que não se restrinjam apenas a panfletos e palestras, e sim em um diálogo que promova a aproximação equipe/usuário ${ }^{(22)}$.

É de fundamental importância o conhecimento sobre a TB, o acesso às informações através dos meios de comunicação, das escolas e de intervenções educativas com o intuito de reduzir o estigma e o impacto das consequências sociais da TB, como também a idealização de programas de educação continuada em saúde, projetados para aumentar o nível de conhecimento em toda a população e como forma de melhorar o acesso dos doentes aos serviços de saúde para o diagnóstico precoce da doença ${ }^{(23)}$.

\section{CONCLUSÃO}

A partir deste estudo, foi possível descrever os componentes ligados à capacitação em TB., que tornam as equipes de saúde capazes de identificar estratégias de cuidado na visão dos profissionais da APS.

Os profissionais tinham acesso ao manual de diretrizes para o controle da TB, fazendo parte da prática cotidiana, o que favorecia no repasse de informações a todos os usuários da Unidade de Saúde, incluindo o papel de cada indivíduo na prevenção e tratamento da TB. Em contrapartida, observouse que ainda há um déficit no requisito capacitação, sendo realizada esporadicamente. Observou-se, também, déficit relacionado ao apoio de especialistas em TB para as equipes da APS.

O manejo de diretrizes e protocolos, a participação de especialistas e de educadores em saúde constituem elementos importantes para as equipes de saúde identificarem estratégias efetivas de cuidado.

Ressalta-se ainda a importância do investimento, por parte das esferas do governo, em capacitações periódicas para os profissionais da APS, em ações de controle a TB, com o intuito de melhorar a assistência prestada aos portadores desta patologia.

Como limitação do estudo, destaca-se a diferença do quantitativo de profissionais por categoria profissional o que impediu inferência à população por categorias.

\section{APOIO FINANCEIRO}

Edital Chamada MCTI/CNPq/MS-SCTIE-Decit Nº 40/2012 - Pesquisa em Doenças Negligenciadas e FAPESP processo número: 2013/22512-3.

\section{- REFERÊNCIAS}

1. Barrêto AJR, Evangelista ALF, de Sá LD, de Almeida SA, Nogueira JA, Lopes AMC. Gestão do cuidado à tuberculose: da formação à prática do enfermeiro. Rev. bras. enferm. [Internet] 2013; 66(6) [acesso em 05 jun 2014]. Disponível: http://dx.doi.org/10.1590/S0034-71672013000600006.

2. Ferreira JCOA, Kurcgant P. Capacitação profissional do enfermeiro de um complexo hospitalar de ensino na visão de seus gestores. Acta paul. enferm. [Internet] 2009; 22(1) [acesso em 10 jan 2016]. Disponível: 
http://dx.doi.org/10.1590/S0103-21002009000100005.

3. Monroe AA, Gonzales RIC, Palha PF, Sassaki CM, Ruffino Netto A, Vendramini SHF, et al. Envolvimento de equipes da atenção básica à saúde no controle da tuberculose. Rev. esc. enferm. USP. [Internet] 2008; 42(2) [acesso em 20 set 2014]. Disponível: http://dx.doi.org/10.1590/S0080-62342008000200008.

4. dos Santos TMMG, Nogueira LT, Arcêncio RA. Atuação de profissionais da Estratégia Saúde da Família no controle da tuberculose. Acta paul. enferm. [Internet] 2012; 25(6) [acesso em 18 ago 2014]. Disponível: http://dx.doi.org/10.1590/S0103-21002012000600020.

5. Silva Sobrinho RA, Souza AL, Wysocki AD, Silva LMC, Beraldo AA, Villa TCS. Conhecimento de enfermeiros de Unidades de Atenção Básica acerca da tuberculose. Cogitare Enferm. [Internet] 2014; 19(1) [acesso em 23 mar 2016]. Disponível: http://dx.doi.org/10.5380/ce.v19i1.35930.

6. World Health Organization (WHO). Global tuberculosis report 2012. Geneva: WHO; 2012.

7. Ministério da Saúde (BR). Secretaria de Vigilância em Saúde. Boletim Epidemiológico. [Internet] 2014; 45(2) [acesso em 16 maio 2014]. Disponível:

http://bvsms.saude.gov.br/bvs/periodicos/boletim_epidemiologico_numero_2_2014.pdf.

8. de Macêdo NB, de Albuquerque PC, de Medeiros KR. O desafio da implementação da educação permanente na gestão da educação na saúde. Trab. educ. saúde. [Internet] 2014; 12(2) [acesso em 07 abr 2014]. Disponível: http://dx.doi.org/10.1590/S1981-77462014000200010.

9. Brasil. Instituto Brasileiro de Geografia e Estatística (IBGE). Estimativas das populações residentes, segundo município. [Internet] 2014 [acesso em 06 maio 2015]. Disponível:

http://www.ibge.gov.br/home/estatistica/populacao/estimativa2014/estimativa_dou.shtm.

10. Natal. Secretaria Municipal de Saúde. Plano municipal de saúde: 2006-2009. [Internet] 2006 [acesso em 06 maio 2015]. Disponível: http://www.natal.rn.gov.br/sms/paginas/File/SMS-PMS2006-2009.pdf.

11. Natal. Sistema de Informação de Agravos de Notificação. Departamento de vigilância à saúde. Setor de vigilância epidemiológica. Núcleo de agravos notificáveis, 2013.

12. Moysés ST, Kusma SZ, Schwab GL. Validação transcultural dos instrumentos ACIC E PACIC para avaliação da percepção das equipes e das pessoas usuárias sobre a atenção às condições crônicas. In: Moysés ST, Silveira F, Antonio D, Moysés SJ. Organizadores. Laboratório de inovações no cuidado das condições crônicas na APS: a implantação do Modelo de Atenção às Condições Crônicas na UBS Alvorada em Curitiba, Paraná. Brasília: Organização Pan-Americana da Saúde/ Conselho Nacional de Secretários de Saúde; 2012. p.81-101.

13. Ministério da Saúde (BR). Conselho Nacional de Saúde. Diretrizes e normas regulamentadoras de pesquisa envolvendo seres humanos. Resolução n. 466, de 12 de dezembro de 2012. Brasília; 2012.

14. Pinto ESG, de Menezes RMP, Villa TCS. Situação de trabalho dos profissionais da Estratégia Saúde da Família em Ceará-Mirim. Rev. esc. enferm. USP. [Internet] 2010; 44(3) [acesso em 08 mar 2015]. Disponível:

http://dx.doi.org/10.1590/S0080-62342010000300015.

15. Ponce MAZ, Wysocki AD, Scatolin BE, Andrade RLP, Arakawa T, Ruffino Netto A, et al. Diagnóstico da tuberculose: desempenho do primeiro serviço de saúde procurado em São José do Rio Preto, São Paulo, Brasil. Cad. Saúde Pública. [Internet] 2013; 29(5) [acesso em 30 nov 2014]. Disponível:

http://dx.doi.org/10.1590/S0102-311X2013000500012.

16. Ministério da Saúde (BR). Manual de recomendações para o controle da tuberculose no Brasil. [Internet] Brasília: Ministério da Saúde; 2011 [acesso em 25 mar 2016]. Disponível:

http://bvsms.saude.gov.br/bvs/publicacoes/manual_recomendacoes_controle_tuberculose_brasil.pdf.

17. Cardoso GCP, da Cruz MM, de Abreu DMF, Decotelli PV, Chrispim PPM, Borenstein JS, et al. A conformidade das ações do tratamento diretamente observado para tuberculose na perspectiva dos profissionais de duas unidades de saúde da cidade do Rio de Janeiro. Cad. Saúde Colet. [Internet] 2012; 20(2) [acesso em 20 nov 2014]. Disponível: http://www.cadernos.iesc.ufrj.br/cadernos/images/csc/2012_2/artigos/csc_v20n2_203-210.pdf.

18. Caliari JS, de Figueiredo RM. Tuberculose: perfil de doentes, fluxo de atendimento e opinião de enfermeiros. Acta paul. enferm. [Internet] 2012; 25(1) [acesso em 11 out 2014]. Disponível: 
http://dx.doi.org/10.1590/S0103-21002012000100008.

19. Hino P, Takahashi RF, Bertolozzi MR, Egry EY. As necessidades de saúde e vulnerabilidades de pessoas com tuberculose segundo as dimensões acesso, vínculo e adesão. Rev. esc. enferm. USP. [Internet] 2011; 45(n. esp2) [acesso em 13 nov 2014]. Disponível: http://dx.doi.org/10.1590/S0080-62342011000800003.

20. Pinto ESG, Scatolin BE, Beraldo AA, Andrade RLP, Silva Sobrinho RA, Villa TCS. O Agente Comunitário de Saúde na detecção de casos de tuberculose. Ciênc. Cuid. Saúde. [Internet] 2014; 13(3) [acesso em 08 mar 2015]. Disponível: http://dx.doi.org/10.4025/cienccuidsaude.v13i3.20926.

21. da Silva EM, Assis MMA, Villa TCS, Scatena LM. Coordenação dos serviços de atenção primária em saúde no controle da tuberculose em um município da Bahia, Brasil. Rev. Baiana de Saúde Pública. [Internet] 2010; 34(2) [acesso em 01 dez 2014]. Disponível: http://inseer.ibict.br/rbsp/index.php/rbsp/article/viewFile/30/30.

22. Alves RS, de Souza KMJ, de Oliveira AAV, Palha PF, Nogueira JA, de Sá LD. Abandono do tratamento da tuberculose e integralidade da atenção na estratégia saúde da família. Texto Contexto Enferm. [Internet] 2012; 21(3) [acesso em 19 jan 2015]. Disponível: http://dx.doi.org/10.1590/S0104-07072012000300021.

23. Beraldo AA, Arakawa T, Pinto ESG, Andrade RLP, Wysocki AD, Silva Sobrinho RA, et al. Atraso na busca por serviço de saúde para o diagnóstico da tuberculose em Ribeirão Preto (SP). Ciênc. saúde coletiva. [Internet] 2012; 17(11) [acesso em 09 fev 2015]. Disponível: http://dx.doi.org/10.1590/S1413-81232012001100024. 